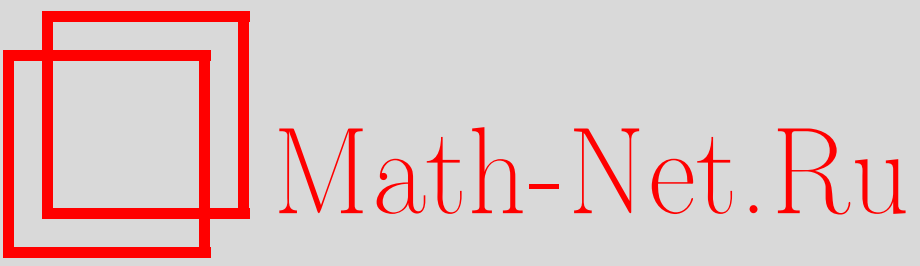

И. А. Истомин, О. Л. Котляров, А. Ю. Лоскутов, К проблеме обработки временных рядов: расширение возможностей метода локальной аппроксимации посредством сингулярного спектрального анализа, ТМФ, 2005, том 142, номер 1, $148-$ 159

DOI: https://doi.org/10.4213/tmf1771

Использование Общероссийского математического портала Math-Net.Ru подразумевает, что вы прочитали и согласны с пользовательским соглашением http: //www . mathnet.ru/rus/agreement

Параметры загрузки:

IP : 54.164 .48 .24

26 апреля 2023 г., 15:32:43 
ТЕОРЕТИЧЕСКАЯ

И МАТЕМАТИЧЕСКАЯ

ФИЗИКА

Том 142, № 1

январь, 2005

(C) 2005 г. И.А. Истомин ${ }^{*}$ О. Л. Котляров* , А. Ю. Лоскутов*

\section{К ПРОБЛЕМЕ ОБРАБОТКИ ВРЕМЕННЫХ РЯДОВ: РАСШИРЕНИЕ ВОЗМОЖНОСТЕЙ МЕТОДА ЛОКАЛЬНОЙ АППРОКСИМАЦИИ ПОСРЕДСТВОМ СИНГУЛЯРНОГО СПЕКТРАЛЬНОГО АНАЛИЗА}

Представлены алгоритмы методов сингулярного спектрального анализа и локальной аппроксимации, применяемых для экстраполяции временно́го ряда. Проанализированы достоинства и недостатки этих методов, рассмотрены особенности их применения для ряда систем. На основе проведенного анализа предложено обобщение метода локальной аппроксимации, позволяющее использовать его для прогноза сильно зашумленных временны́х рядов. Приведены результаты численных расчетов, иллюстрирующие возможности предлагаемого метода.

Ключевые слова: временные ряды, прогноз, хаос, локальная аппроксимация.

\section{1. ВВЕДЕНИЕ}

Анализ результатов любого эксперимента основывается на обработке полученных данных. Во многих случаях эти данные представляют собой временны́е ряды - расположенные в хронологическом поря дке последовательности значений одной или нескольких измеряемых величин. Обработка временны́х рядов с целью извлечения из них полезной информации о свойствах системы - задача сама по себе очень важная и интересная. Однако во многих случаях основное внимание уделяется не исследованию свойств самой системы, а прогнозу динамики порожденного ею временно́го ряда. Например, в метеорологии практический интерес представляет в первую очередь прогноз погоды на ближайшее время, тогда как более глобальная задача изучения особенностей климата имеет в краткосрочной перспективе несколько меньшее значение. Таким образом, помимо исследования свойств системы, породившей временно́й ряд, не менее важным бывает прогноз ее дальнейшей траектории.

Задача прогноза не является исключительной принадлежностью метеорологии, она также актуальна в геофизике, где предсказание землетрясений является одним из основных направлений исследований, в астрофизике при изучении солнечной активности,

* Московский государственный университет, Москва, Россия. E-mail: Loskutov@moldyn.phys.msu.ru 
финансовом анализе при прогнозе курсов акций, биржевых индексов и т.д. В этих и других областях исследований уже достаточно давно применяются методы прогноза (или экстраполящии) временны́х рядов, относяшиеся к классу ARMA (AutoRegressive Moving Average) [1], [2]. Их основная идея - выражение следующих значений ряда через предыдущие. Это наиболее распространенный подход, используемый в ситуациях, когда никакой другой информации о системе, кроме заключенной в предыдущих значениях ряда, нет.

Специальные методы прогноза, основанные на работах Такенса [3], также разрабатывались в рамках теории динамических систем. Они ориентированы, в первую очередь, на прогнозирование нерегулярных (хаотических и квазипериодических) стационарных временны́х рядов, порождаемых сложными нелинейными системами. Однако с развитием нелинейной динамики оказалось, что задача прогноза значительно сложнее и часто не укладывается в схемы, представляемые теорией. В частности, длина обрабатываемого ряда должна быть достаточно большой, а шумовая компонента-малой величиной.

Один из эффективных способов, позволяющих исследовать зашумленные ряды относительно небольшой длины, предложен в данной работе. В его основе лежит метод локальной аппроксимации (ЛА), описанный впервые в статье [4] для прогноза хаотических временнь́х рядов. В оригинальном варианте этот метод имеет ряд преимушеств перед традиционным методом авторегрессии при прогнозировании нерегулярных временны́х рядов, однако он пока не получил широкого распространения в основном из-за трудностей при анализе коротких и зашумленных временнь́хх рядов.

В настояшей работе рассматривается возможность повышения эффективности метода ЛА при прогнозировании зашумленных временны́х рядов посредством предварительной фильтрации данных с помощью сингулярного спектрального анализа (CCA) [5]. Метод ССА разработан в рамках нелинейной динамики для обработки временни́х рядов, однако используется главным образом для определения их основных составляющих [6] и подавления шума [7], хотя имеются и основанные на нем оригинальные алгоритмы прогноза [8].

Сейчас существует много методов обработки временни́х рядов. Это вейвлет-анализ, фликкер-шумовая спектроскопия и др. (см., например, [9]). Все они достаточно эффективны и позволяют обрабатывать, а также в той или иной степени предсказывать динамику систем. Предлагаемое в данной работе обобщение дает возможность осушествлять прогнозирование хаотических и квазипериодических рядов в присутствии случайных шумовых компонент.

\section{2. МЕТОДЫ ОБРАБОТКИ ВРЕМЕННЫХ РЯДОВ}

2.1. Метод задержек. В основе большинства подходов, связанных с обработкой временны́х рядов $\left\{x_{1}, \ldots, x_{N}\right\}$, лежит построение множества векторов задержек $\mathbf{z}_{i}=$ $\left(x_{i}, x_{i+1}, \ldots, x_{i+m}\right)^{\mathrm{T}}[10]$. В методах анализа временни́х рядов, разработанных в рамках нелинейной динамики, это также является первым и необходимым шагом.

Метод задержек устанавливает переход от исходного одномерного (скалярного) временно́го ряда к многомерному (векторному) представлению, сходному с используемым в авторегрессии. При этом каждый многомерный вектор образуется из некоторого числа $m$ следуюших друг за другом значений исходного временно́го ряда. Результат можно 
представить в виде набора “фотографий” ряда, сделанных через скользяшее вдоль ряда окно, в которое одновременно попадает лишь $m$ последовательных значений ряда:

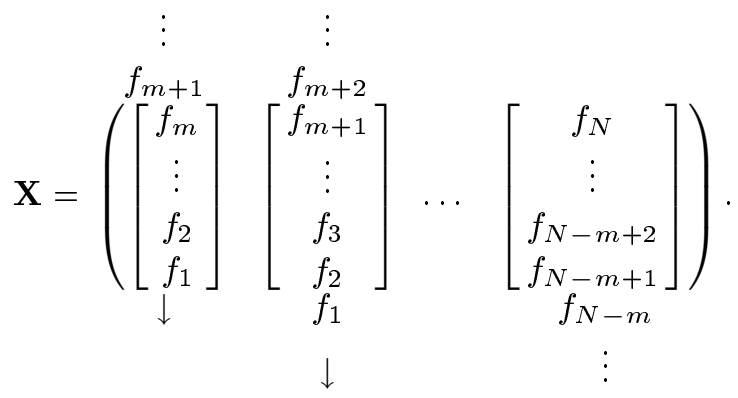

Здесь $f_{1}, f_{2}, \ldots, f_{N}$ - значения ряда в моменты времени $t=1,2, \ldots, N$. Каждая квадратная скобка - вектор в $m$-мерном пространстве задержек; последовательность таких векторов задает матрицу наблюдений $\mathbf{X}_{m \times(N-m+1)}$, где $N$ - число элементов исходного ряда. Эта матрица, в каждом столбце которой стоят части одного и того же ряда, сдвинутые друг относительно друга, и будет многомерным представлением исходного скалярного ряда в пространстве задержек.

Новым результатом явилось установление того, что пространство задержек при соблюдении определенных условий может рассматриваться как реконструкция фазового пространства нелинейной динамической системы, породившей временной ряд (теорема Такенса и ее обобщения [3], [11]). Таким образом, была доказана возможность описания динамики многомерной системы по временно́му ряду наблюдаемой. В свою очередь, возможность описания и реконструкции динамики системы при определенных условиях позволяет прогнозировать ее дальнейшее поведение [12].

Определение размерности реконструкции. Переход к многомерному представлению в рассматриваемом нами дискретном случае задается одним параметром - размерностью пространства задержек или размерностью вложения (реконструкции) $m$. От правильного выбора этой величины во многом зависит возможность получения точного и надежного прогноза. Ограничение $m \geqslant 2 d+1$, где $d$ - размерность породившей ряд системы [3], [11], редко помогает в выборе размерности реконструкции, так как $d$ обычно априори неизвестно, а определить размерность системы на основе имеющихся данных достаточно сложно, особенно в случае коротких и сильно зашумленных рядов. Наиболее популярным алгоритмом для оценки размерности вложения (и размерности системы) на сегодняшний день является алгоритм Грассбергера-Прокачиа [13], [14], но и он оказывается не очень эффективным при работе с короткими (до $10^{4}$ значений) временны́ми рядами.

Указанные сложности в определении размерности реконструкции часто преодолеваются в рамках применяемого метода прогноза. При этом имеющийся ряд разбивается на две неравные следуюшие друг за другом части, и меньшая из них используется для проверки качества прогноза, сделанного по другой части. Размерность, при которой получается лучший прогноз, считается оптимальной для данного ряда.

2.2. Метод локальной аппроксимации. Как отмечалось выше, в настояшее время для прогноза временни́х рядов чаше всего используются методы авторегрессии, при- 
надлежащие к классу методов ARMA $[1]^{1)}$. Модель авторегрессии порядка $p$ имеет вид

$$
f_{t}=a_{0}+a_{1} f_{t-1}+a_{2} f_{t-2}+\cdots+a_{p} f_{t-p}+\xi_{t}
$$

где $f_{t}$ - значение ряда на шаге $t,\left\{\xi_{t}\right\}$ - последовательность случайных величин, образуюшая белый шум. В этом случае, для того чтобы предсказывать дальнейшую траекторию ряда, надо сначала определить порядок авторегрессии, а затем на основе имеющихся данных получить оценки коэффициентов авторегрессии $\left\{a_{0}, \ldots, a_{p}\right\}$. При этом выбор порядка авторегрессии осушествляется, как правило, исходя из вида частной автокорреляционной функции. Коэффициенты оцениваются по всем имеюшимся данным и, соответственно, предполагаются не зависящими от времени. Таким образом, данная аппроксимация является глобальной.

Методы глобальной линейной аппроксимации дают хорошее приближение функции до тех пор, пока достаточно свободных параметров, а данных хватает на устойчивую оценку этих параметров. Однако если функция довольно сложная, то нет никакой гарантии, что можно найти такое представление, которое позволит эффективно ее аппроксимировать. Может получиться замкнутый круг: чем сложнее функция, тем больше надо параметров, чем больше параметров необходимо оценить, тем больше надо данных, а увеличение количества используемых данных может потребовать введения в модель дополнительных параметров и т.д.

Один из способов избежать этого зацикливания - воспользоваться методом ЛА. Основная его идея состоит в том, чтобы разбить всю область определения функции на несколько локальных областей и строить аппроксимируюшие модели и оценивать параметры этих моделей отдельно в каждой области. Области должны быть достаточно малыми, чтобы функция в каждой из них не изменялась “слишком резко”. Это позволяет применять в каждой области достаточно простые модели, например линейные. Главное условие эффективного применения метода ЛА - удачный выбор размера локальной области, или, что практически то же самое, числа соседей. Это число надо выбрать так, чтобы в каждой области их было достаточно для устойчивой оценки параметров, а добавление небольшого числа новых соседей не давало сушественного изменения оцениваемых параметров.

Метод ЛА [4] стал первым локальным методом, разработанным для прогноза временны́х рядов и опирающимся на теорему Такенса. Этот метод также использует представление типа (2), но при этом имеет два основных отличия от метода авторегрессии:

1) коэффициенты в выражении (2) оцениваются отдельно для каждой из локальных областей;

2) выражение (2) может включать и нелинейные члены, т.е. различные степени значений ряда в предшествуюшие моменты (максимальную степень называют порядком аппроксимации).

В качестве величины, имеюшей смысл порядка авторегрессии, используется размерность реконструкции.

\footnotetext{
1) В последнее время с развитием вычислительных средств для построения прогнозов все чаще применяются нейронные сети, однако по частоте и широте использования у метода ARMA пока нет конкурентов.
} 
Следовательно, метод ЛА оказывается более обоснованным в выборе “порядка авторегрессии" и более гибким в использовании исходных данных за счет применения различных наборов коэффициентов на различных участках траектории. Таким образом, появляется возможность аппроксимации существенно нелинейных функций посредством набора кусочно-линейных аппроксимаций.

Остановимся кратко на основных шагах алгоритма, реализуюшего метод ЛА.

1. Выбор локального представления. Этот шаг включает в себя вычисление размерности реконструкции и построение многомерного представления ряда - матрицы задержек $\mathbf{X}$.

2. Определение окрестности или числа соседей. Как правило, окрестность задается выбором числа соседей $N^{s}$. Для этого в пространстве задержек (среди столбцов матрицы $\mathbf{X}$ ) выбираются наиболее "близкие". В качестве самого простого критерия близости можно выбрать следующий. Для данной метрики $\|\cdot\|$ и при заданном числе соседей $N^{s}$ набор $\left\{\mathbf{y}^{t}\right\}$ будет набором ближайших соседей $\mathbf{x}$, если он обеспечивает минимум суммы $\sum_{t=1}^{N^{s}}\left\|\mathbf{y}^{t}-\mathbf{x}\right\|$. Здесь стоит отметить, что, хотя и рассматривается динамика изменения наблюдаемой, близость $\mathbf{y}^{t}$ к значениям $\mathbf{x}$ не подразумевает близость во времени.

3. Выбор модели аппроксимации и ее идентификация. На этом шаге при уже определенной размерности реконструкции выбирается порядок аппроксимации (обычно используется либо линейная аппроксимация, либо квадратичная по значениям ряда в предшествуюшие моменты времени):

$$
x_{1}^{t+T}=f^{t+T}\left(\mathbf{x}^{t}\right)=\mathbf{P}_{q}\left(x_{1}^{t}, x_{2}^{t}, \ldots, x_{m}^{t}\right)
$$

где $T=1,2, \ldots, q$ - степень полинома, т.е. порядок аппроксимации. Поэтому схемы ЛА можно классифицировать в соответствии с порядком аппроксимируюшего полинома. Коэффициенты модели обычно оцениваются методом наименьших квадратов с помощью так называемого сингулярного разложения [15]. Подробное изложение и сравнение способов вычисления коэффициентов модели при применении ЛА дано в работе [16].

В некоторых случаях при сильно ограниченной длине ряда используется аппроксимация нулевого порядка, т.е. принимается гипотеза об отсутствии явной зависимости следуюших значений ряда от предыдуших внутри каждой локальной области. В этом случае "прогноз" зависит лишь от попадания в конкретную локальную область. Такую ситуацию хорошо иллюстрирует пример, связанный с прогнозированием погоды [17]: один из возможных способов построения прогноза температуры на следующий день состоит в том, чтобы, выбрав, в какой из предшествуюших дней температура была максимально близкой к сегодняшней, взять в качестве прогноза температуры на завтра ее величину в следующий за найденным день. Аппроксимация более высокого порядка позволяет также учитывать влияние на прогноз отклонений сегодняшней температуры от температуры в выбранный день.

4. На последнем шаге строится прогноз для следуюшего значения ряда в предположении, что эволюция последнего члена ряда происходит по тому же закону, что у других векторов из его локальной окрестности.

Способы экстраполящии. Для построения прогноза на несколько шагов вперед используются два основных способа экстраполяции [17]. В соответствии с первым, итеративнылм, способом оцениваются параметры аппроксимирующей модели (3) для $T=1$. 
Дальнейший прогноз для $T=2,3, \ldots$ представляет собой последовательность итераций, т.е. предсказанное значение добавляется к исходному ряду. Далее предполагается, что получившийся новый вектор в пространстве задержек попадает в ту же окрестность (т.е. эволюционирует по тому же закону) и строится прогноз еше на один шаг вперед, и т.д. Как показано в работе [18], последняя гипотеза может приводить к систематической ошибке прогноза.

Альтернативный прямой прогноз состоит в оценивании параметров отдельно для каждого значения $T$. Этот способ позволяет строить более точный прогноз, так как в этом случае не происходит накопления ошибки на каждом шаге [19].

Влияние шума. При разработке метода ЛА предполагалось, что точность предсказаний определяется качеством аппроксимации, которая, в свою очередь, зависит от объема имеющихся данных. Однако, естественно, во многих случаях точность предсказания может быть ограничена также и шумом: даже если мы знаем уравнения движения точно, шум сужает пределы предсказуемости, внося ошибку в определение начальных условий и искажая детерминированные траектории. В работе [17] показано, что влияние шума на качество прогноза очень похоже по последствиям на влияние ошибок аппроксимации. В методе ЛА наиболее серьезной ошибкой, возникаюшей под влиянием шума, является ошибка в выборе соседей.

2.3. Сингулярный спектральный анализ. Метод ССА разрабатывался, в первую очередь, как метод выделения из ряда периодических и квазипериодических составляюших. Также было показано, что этот метод можно использовать и для улучшения отношения сигнал/шум [7]. В последнее время появились варианты, расширяющие возможности ССА и позволяющие строить на его основе прогноз дальнейшей динамики ряда [8], [20]. В настоящей работе этот метод используется исключительно как средство подавления шума.

Основная идея метода ССА состоит в обработке матрицы $\mathbf{X}$ по алгоритму, близкому к методу главных компонент (ГК). Использование метода ГК является наиболее важным положением метода ССА, отличающим его от других методов нелинейной динамики, применяемых для анализа и прогноза временни́х рядов. Суть метода ГК состоит в снижении размерности исходного пространства факторов (у нас это пространство задержек) посредством перехода к более “информативным" переменным (координатам). Полученные таким образом новые переменные и называют ГК. Этот переход осушествляется с помощью ортогонального линейного преобразования.

На практике задача перехода к ГК сводится к поиску собственных векторов и собственных значений матрицы $\mathbf{X} \cdot \mathbf{X}^{\mathrm{T}}[21]$. Пусть

$$
\boldsymbol{\Lambda}=\left(\begin{array}{cccc}
\lambda_{1} & 0 & \ldots & 0 \\
0 & \lambda_{2} & \ldots & 0 \\
\vdots & \vdots & \ddots & \vdots \\
0 & 0 & \ldots & \lambda_{M}
\end{array}\right)
$$

и $\mathbf{V}_{M \times M}$ - матрица собственных значений и матрица собственных векторов $\mathbf{X} \cdot \mathbf{X}^{\mathrm{T}}$, соответственно. Поскольку размерности реконструкции (и соответственно матрицы $\mathbf{X}$ ), применяемые в ЛА и ССА, в обшем случае могут различаться, для ССА размерность 
реконструкции будем обозначать символом $M$. При этом матрица ГК имеет вид $\mathbf{Z}=$ $\mathbf{V}^{\mathrm{T}} \cdot \mathbf{X}$. При таком преобразовании ГК представляют собой совокупности проекций точек исходного набора на собственные векторы. Величины собственных значений характеризуют разброс точек вдоль новых осей координат. Максимальным является первое собственное значение, а следуюшие монотонно убывают.

ГК обладают многими полезными свойствами. В ССА получающееся разложение используется для выделения наиболее значимых составляюших ряда и отсева случайных возмушений. Основная идея этой фильтрации состоит в использовании для реконструкции матрицы $\mathbf{X}$ не всех $\Gamma \mathrm{K}$, а лиш $r$ наиболее значимых.

В обшем случае аппроксимация матрицы $\mathbf{X}$ получается как

$$
\widehat{\mathbf{X}}=\mathbf{V}_{M \times r} \cdot \mathbf{V}_{M \times r}^{\mathrm{T}} \cdot \mathbf{X}
$$

где $\mathbf{V}_{M \times r}$ - часть матрицы собственных векторов (первые $r$ столбцов), соответствующая $r$ первым ГК. Следующий шаг после аппроксимации матрицы $\mathbf{X}$ - восстановление исходного ряда. При реконструкции по первым ГК матрища $\mathbf{X}$ теряет свой первоначальный диагональный вид, поэтому необходимо провести усреднение вдоль всех диагоналей, на которых изначально стояли одинаковые значения:

$$
\hat{f}_{t}= \begin{cases}\frac{1}{t} \sum_{i=1}^{t} \hat{x}_{M-t+i, t}, & t<M, \\ \frac{1}{M} \sum_{i=1}^{M} \hat{x}_{M-t+i, t}, & M \leqslant t \leqslant N-M+1, \\ \frac{1}{N-t+1} \sum_{i=1}^{N-t+1} \hat{x}_{i, t-M+i}, & N-M+1 \leqslant t \leqslant N .\end{cases}
$$

Таким образом, алгоритм реконструкции временно́го ряда методом ССА включает в себя три основных этапа:

1) построение матрицы $\mathbf{X}$;

2) вычисление ГК и выбор наиболее значимых из них;

3) восстановление ряда по выбранным ГК.

Применение этого алгоритма позволяет сгладить исходный ряд, снизить уровень случайных возмущений, повысить отношение сигнал/шум. Однако разработанные на его основе методы прогнозирования [8] оказываются недостаточно эффективными при работе с непериодическими рядами.

Так же как и в методе ЛА, в ССА существует несколько модификаций. Они связаны в основном с предварительным центрированием и/или нормированием строк матрицы $\mathbf{X}$. Мы будем использовать вариант с центрированными строками как наиболее близкий к стандартному методу ГК.

\section{3. ОБОБШЕНИЕ МЕТОДА ЛА}

3.1. Алгоритм ССА-ЛА. В случае сильно зашумленных рядов даже увеличение числа наблюдений не позволяет эффективно применять алгоритм ЛА, так как в этом случае высока вероятность появления большого количества ложных соседей и отсева 
соседей истинных. Поэтому представляется целесообразным для улучшения качества прогноза соединить оба рассмотренных метода (ССА и ЛА) в один, в котором метод ССА использовался бы только для фильтрации исходного временно́го ряда (подавления шума), а сам прогноз строился бы по методу ЛА.

Таким образом, предлагаемый метод (назовем его ССА-ЛА) можно рассматривать как расширение метода ЛА, позволяюшее применять его к сильно зашумленным временни́м рядам. В ближайшее время планируется также исследовать возможность выделения тренда и периодических составляющих в процессе ССА-фильтрации, что особенно важно в экономических временнь́х рядах.

Полностью алгоритм ССА-ЛА состоит из двух основных этапов:

1) ССА-фильтрация, т.е. подавление шума;

2) прогнозирование сглаженного ряда методом ЛА.

Алгоритмы методов ССА и ЛА были описаны выше. Здесь же отметим, что на основе специального анализа [19] для построения прогноза был выбран прямой вариант ЛА первого порядка и ССА с центрированием.

При совместном применении методов ССА и ЛА выяснилось, что $M$ первых и $M$ последних по времени значений ряда реконструируются со значительными отклонениями от исходных значений, тогда как остальная часть ряда приближается намного точнее. По-видимому, снижение точности аппроксимации граничных значений есть следствие более короткого интервала усреднения, используемого при их вычислении в (5). Поэтому при построении прогноза $M$ первых и $M$ последних значений выровненного ряда отбрасывались и прогноз строился начиная с $(N-M+1)$-го значения.

3.2. Численные результаты. Представим некоторые численные результаты, на основе которых можно оценить возможности применения метода ССА-ЛА для прогноза сильно зашумленных нерегулярных временнь́х рядов. Такие временнь́е ряды были получены из дифференциально-разностного уравнения Макки-Гласса [22], которое часто используется в качестве тестового примера (см. [14], [17]):

$$
\frac{d x}{d t}=\frac{a x(t-\Delta T)}{1+x^{c}(t-\Delta T)}-b x(t),
$$

где $a=0.2, b=0.1, c=10, \Delta T=17$. Кроме того, мы использовали известную систему Лоренца

$$
\begin{aligned}
& \dot{x}=\sigma(y-x), \\
& \dot{y}=(r-z) x-y, \\
& \dot{z}=x y-b z,
\end{aligned}
$$

где $\sigma=5, r=15, b=1$. Ряды были рассчитаны один раз для всех численных экспериментов, а шумовая добавка в виде некоррелированного гауссового шума с нулевым математическим ожиданием генерировалась отдельно в каждом опыте.

Результаты анализа представлены на рис. 1-3. Для прогнозирования были рассчитаны ряды каждого вида по 3600 точек каждый, из которых 110 точек использовались 


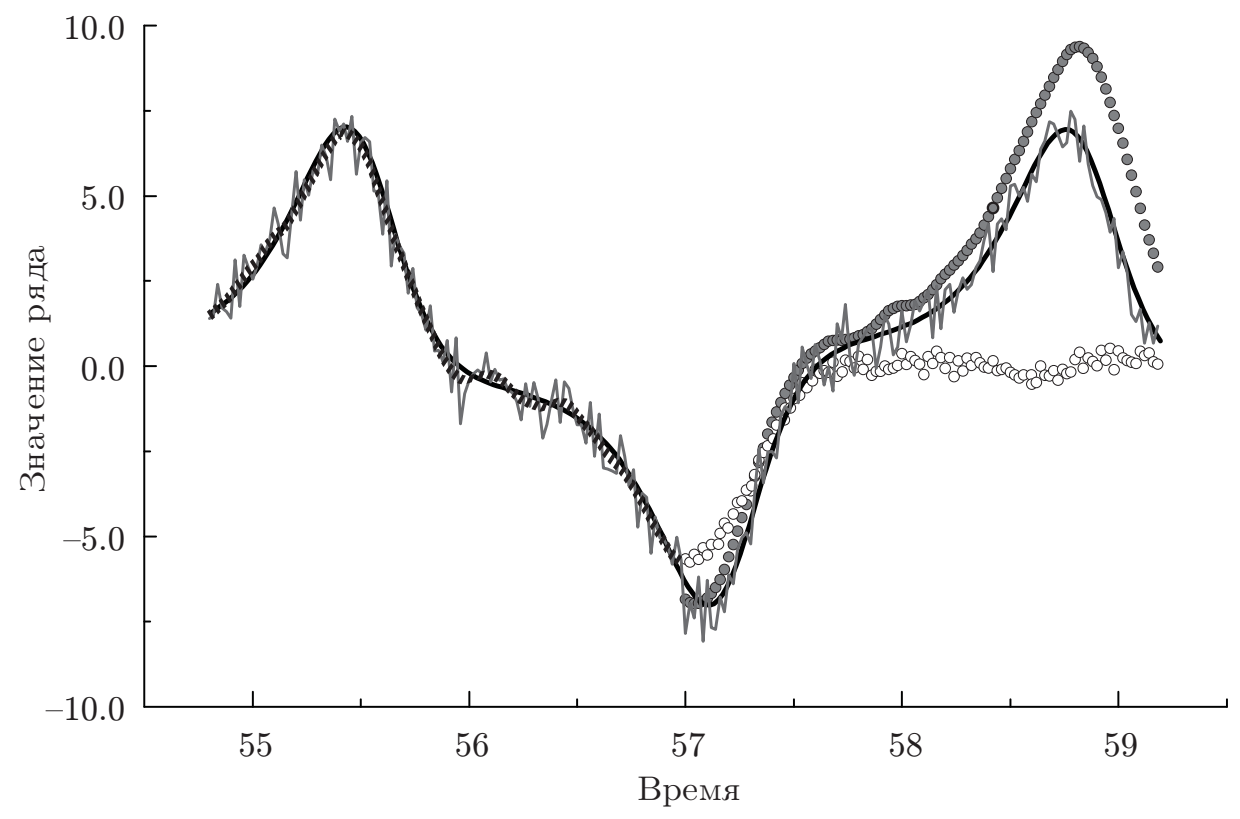

Рис. 1. ССА-фильтрация и прогноз $x$-компоненты системы Лоренца с наложенным пятипроцентным (от максимального значения) гауссовым шумом. Прогноз начинается с момента 57. Длина прогноза - 110 точек. Сплошная линия - исходный ряд; ломаная линия исходный ряд с добавлением шума; пунктир - результат сглаживания зашумленного ряда; пустые кружки - прогноз методом ЛА; заполненные кружки - прогноз методом ССА-ЛА.

только для оценки качества прогноза. Параметры ССА-фильтрации и ЛА были выбраны следуюшие: $M=18, r=6$ и $m=6, N^{s}=21$, соответственно. При этом задача согласования параметров ЛА и ССА на данном этапе решалась эмпирически.

Рассмотрим сначала пример прогнозирования $x$-компоненты системы Лоренца (рис. 1). В левой части графика (до момента 57.0) показан результат ССА-фильтрации зашумленного сигнала (последние 110 точек), который, как видно, является достаточно точной реконструкцией исходного незашумленного ряда. Однако фаза реконструируется с небольшой ошибкой, и это сильнее всего заметно в области максимума. С момента времени 57.0 начинается прогноз. На графике он показан кружочками. Видно, что в этом примере предварительная фильтрация позволила существенно повысить точность прогноза.

Вообще говоря, точность прогноза может сильно зависеть от момента начала прогноза. Для того чтобы получить более объективную оценку качества прогнозов, получаемых обычным ЛА и ССА-ЛА методами, необходимо воспользоваться характеристикой, менее зависящей от точки старта. В качестве такой характеристики для сравнения точности прогнозов, получаемых различными методами (см. [17]), будем использовать величину нормированной среднеквадратичной ошибки или ошибки прогноза:

$$
E=\sqrt{\frac{\left\langle\left(f_{t+T}-\hat{f}_{t+T}\right)^{2}\right\rangle_{t}}{\left\langle\left(f_{t}-\left\langle f_{t}\right\rangle_{t}\right)^{2}\right\rangle_{t}}}
$$


Здесь усреднение проводится по моментам начала прогноза. Естественно, чем меньше ошибка прогноза, тем лучше сам прогноз. Если величина $E$ превышает единицу, то это означает, что прогноз неудачный и вместо него лучше просто взять среднее значение ряда. Для большей надежности получаемой ошибки в соотношении (6) используется не арифметическое среднее по начальным моментам прогноза, а медиана.

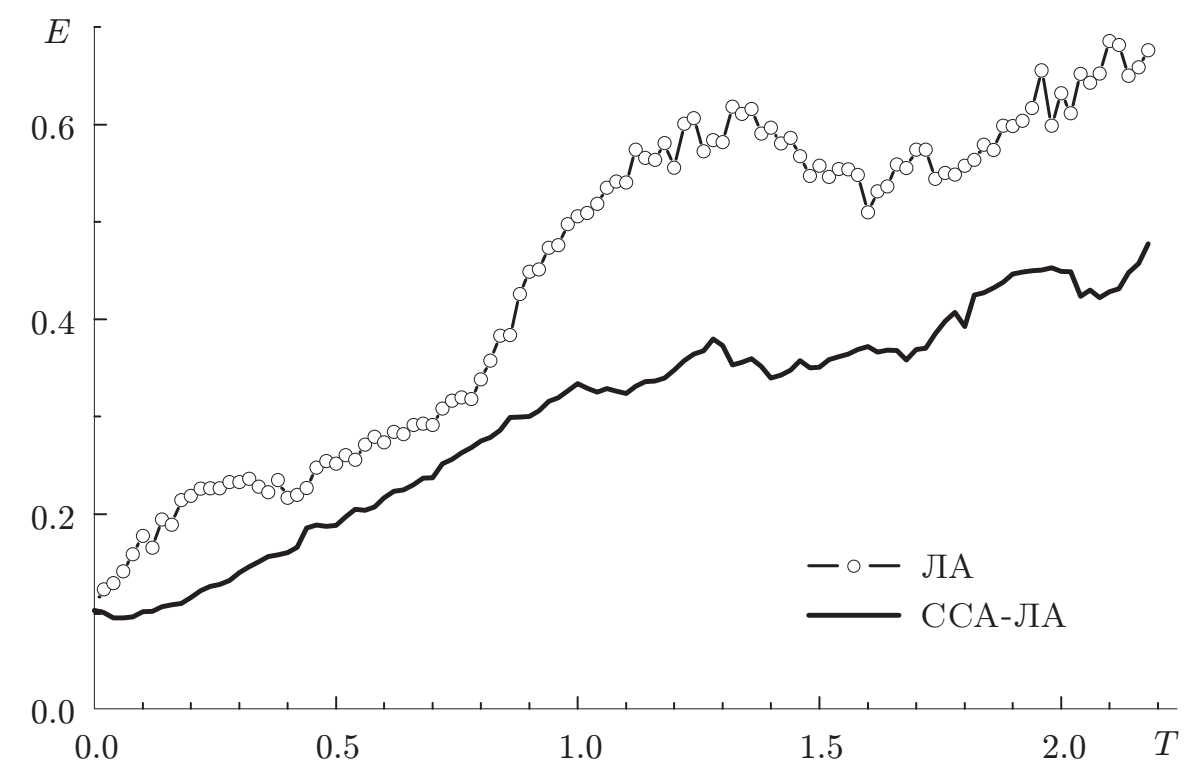

Рис. 2. Ошибка прогноза $x$-компоненты системы Лоренца (прогноз по данным с наложенным пятипроцентным шумом). Медианное усреднение проведено по 500 точкам старта.

Для $x$-компоненты системы Лоренца, которая рассматривалась выше, зависимость ошибки (6) от интервала прогноза показана на рис. 2. Так же как в примере на рис. 1, прогноз с предварительной фильтрацией и в среднем оказался лучше: ошибка прогноза в случае алгоритма ССА-ЛА стабильно меньше ошибки обыкновенного метода ЛА. Зависимости, подобные представленной на рис. 2, строились для различных амплитуд шума и всегда, кроме нулевого уровня, с помощью метода ССА-ЛА получались более точные прогнозы.

По тому же критерию - величине ошибки прогноза - проводилось сравнения и для ряда, полученного на основе уравнения Макки-Гласса. Результаты этого сравнения очень похожи на результаты, полученные для ряда Лоренца. Соответствующие зависимости ошибок прогноза от длины интервала прогнозирования для разных значений шума представлены на рис. 3. Для этого ряда алгоритм ССА-ЛА также оказывается точнее обычного метода ЛА.

Таким образом, результаты численного анализа показывают, что предварительная ССА-фильтрация позволяет значительно повысить точность прогноза, получаемого методом ЛА, и объединение этих двух методов в единый алгоритм ССА-ЛА может быть особенно полезно при прогнозировании зашумленных рядов, с которыми ЛА справляется недостаточно эффективно. 


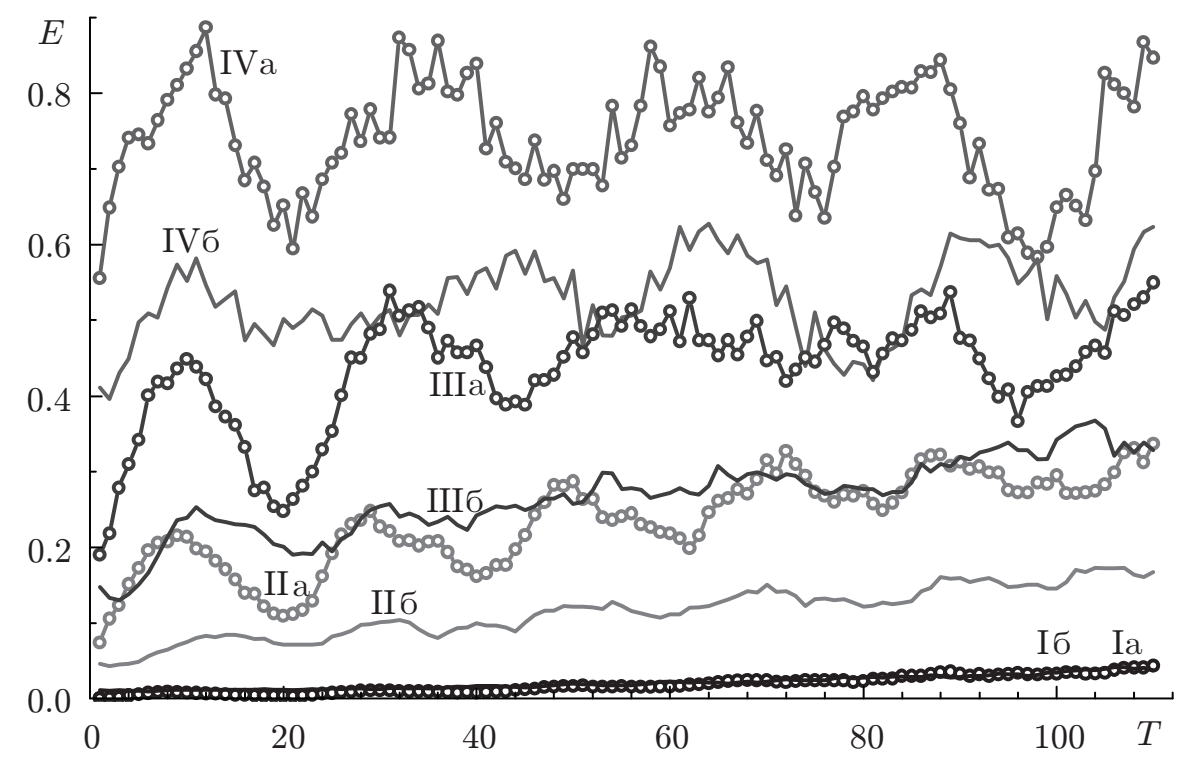

Рис. 3. Ошибка прогноза методами ЛА (а) и ССА-ЛА (б) для уравнения Макки-Гласса в зависимости от интервала прогнозирования в отсутствие шума (I) и при амплитудах шума $1.5 \%$ (II), $5 \%$ (III), $20 \%$ (IV) от максимального значения. Медианное усреднение проведено по 500 стартовым точкам.

\section{4. ЗАКЛЮЧЕНИЕ}

Прогнозирование временны́х рядов методом ЛА является пока скорее принципиальной возможностью, чем реальным инструментом исследований. В то же время алгоритм ЛА имеет несомненное преимушество перед обычной авторегрессией. Оно заключается в использовании кусочно-линейного приближения вместо глобальной линейной аппроксимации, что позволяет с помошью ЛА успешно прогнозировать нерегулярные временны́е ряды, для которых линейное авторегрессионное представление неприемлемо. При этом разбиение на области, внутри которых используется линейная аппроксимация, происходит не по временно́й шкале, а в пространстве задержек. Поэтому в одну область попадают действительно близкие состояния. Это и позволяет применять линейную аппроксимацию внутри каждой области, тогда как глобальная линейная аппроксимация, соответствуюшая аппроксимации всего фазового пространства системы единственной гиперплоскостью, может быть абсолютно нереалистичной.

Основная причина, ограничивающая использование метода ЛА, состоит в том, что его эффективное применение возможно только для прогнозирования достаточно длинных временны́х рядов. При этом в случае сильной зашумленности данных требования к длине исходного ряда, естественно, сушественно возрастают, а для реальных систем редко удается получить ряд необходимой длины. Чтобы ослабить это ограничение, в настоящей работе предлагается использовать предварительную фильтрацию ряда с помощью метода ССА, который хорошо зарекомендовал себя именно как эффективное средство подавления шума, особенно для нерегулярных временнь́х рядов, т.е. в тех случаях, когда не применима фурье-фильтрация. 
Как показали численные исследования, такое сочетание методов ССА и ЛА дает устойчиво более точный прогноз, чем обычный метод ЛА. Более того, результат практически не зависит от уровня шума, длины прогноза и системы, породившей исследуемый временной ряд. Это дает основания считать, что использование ССА-фильтрации как необходимой составной части в алгоритме ССА-ЛА может сушественно расширить область применения метода ЛА и позволит на практике оценить его преимущества перед традиционными методами построения прогноза.

\section{Список литературы}

[1] Дж. Бокс, Г. Дженкинс. Анализ временны́х рядов. Прогноз и управление. М.: Мир, 1974.

[2] А.С. Монин, Л. И. Питербарг. Предсказуемость погоды и климата. В сб.: Пределы предсказуемости. Ред. Ю. А. Кравцов. М.: Наука, 1992. С. 28-53.

[3] F. Takens. Detecting strange attractors in turbulence. In: Dynamical Systems and Turbulence. Lect. Notes in Math. V. 898. Eds. D. A. Rand, L. S. Young. Berlin: Springer, 1981. P. 336-381.

[4] J. D. Farmer, J. J. Sidorowich. Phys. Rev. Lett. 1987. V. 59. P. 845-848.

[5] R. Vautard, P. Yiou, M. Ghil. Physica D. 1992. V. 58. P. 95-126.

[6] А.Ю. Лоскутов, И. А. Истомин, О. Л. Котляров, К. М. Кузанян. Письма в Астрон. журн. 2001. Т. 27. № 11. С. 867-876.

[7] M. Ghil, M.R. Allen, M.D. Dettinger et al. Rev. Geophys. 2002. V. 40(3). P. 1-41.

[8] Д. Л. Данилов, А. А. Жиглявский (ред.). Главные компоненты временных рядов: метод "Гусеница". СПб.: Изд-во СПб ГУ, 1997.

[9] И. М. Дремин, О. В. Иванов, В.А. Нечитайло. УФН. 2001. Т. 171. № 5. С. 465-501; A. V. Descherevsky, A.A. Lukk, A. Sidorin, G.V. Vstovsky, S. F. Timashev. Nat. Hazards and Earth Syst. Sci. 2003. V. 3. № 2. P. 159-164.

[10] D. S. Broomhead, G. P. King. Physica D. 1986. V. 20. P. 217-236.

[11] R. Mañe. On the dimension of the compact invariant sets of certain nonlinear maps. In: Dynamical Systems and Turbulence. Lect. Notes in Math. V. 898. Eds. D. A. Rand, L. S. Young. Berlin: Springer, 1981. P. 230-242.

[12] Г. Г. Малинецкий, А. Б. Потапов. Современные проблемы нелинейной динамики. М.: YPCC, 2000.

[13] P. Grassberger, I. Procaccia. Phys. Rev. Lett. 1983. V. 50. P. 346-349.

[14] P. Grassberger, I. Procaccia. Physica D. 1983. V. 9. № 1, 2. P. 189-201.

[15] Д. Каханер, К. Моулер, С. Нәи. Численные методы и программное обеспечение. М.: Мир, 1998.

[16] D. Kugiumtzis, O. C. Lingjoerde, N. Christophersen. Physica D. 1998. V. 112. P. 344-360.

[17] J. D. Farmer, J. J. Sidorowich. Exploting chaos to predict the future and reduce noise. In: Evolution, Learning, and Cognition. Ed. Y. C. Lee. Singapore: World Scientific, 1988. P. 277-330.

[18] K. Judd, M. Small. Physica D. 2000. V. 136. P. 31-44.

[19] А.Ю. Лоскутов, О.Л. Котляров, И. А. Истомин, Д. И. Журавлев. Вестн. МГУ. Физика, Астрономия. 2002. № 6. С. 3-21.

[20] A. Loskutov, I. A. Istomin, K. M. Kuzanyan, O. L. Kotlyarov. Nonlin. Phenomena in Complex Syst. 2001. V. 4. № 1. P. 47-57.

[21] А. М. Дубров, В. С. Мхитарян, Л. И. Трошин. Многомерные статистические методы. М.: Финансы и статистика, 2000.

[22] M. C. Mackey, L. Glass. Science. 1977. V. 197. P. 287-289.

Поступила в редакцию 11.XI.2003 г., после доработки 3.VI.2004 г. 